\title{
Terapia com exposição à realidade virtual e avaliação funcional para fobia de dirigir: um programa de intervenção
}

\author{
Therapy with Exposure to Uirtual Reality and functional Assessment \\ for Driuing Phobia: An Interuention Program \\ Terapia con exposición a la realidad uirtual y evaluación funcional \\ para la fobia a conducir: un programa de interuención
}

\author{
Andressa dos Santos** \\ Universidade Estadual de Londrina \\ Elizeu Borloti* \\ Universidade Federal do Espírito Santo \\ Verônica Bender Haydu** \\ Universidade Estadual de Londrina
}

Doi: http://dx.doi.org/10.12804/revistas.urosario.edu.co/apl/a.5329

\section{Resumo}

Este estudo avaliou os efeitos de um programa de intervenção no medo de dirigir. Tal estudo incluiu a avaliação: da função de comportamentos de participantes com medo de dirigir; da exposição à realidade virtual; da capacidade do simulador de gerar senso de presença e ansiedade; e do cybersickness. Participaram adultos com queixa de medo de dirigir, com idades entre 22 e 54 anos, portadores de Carteira Nacional de Habilitação. Um simulador de direção veicular foi usado. O procedimento consistiu de um delineamento de linha de base múltipla não simultânea, com 11 a 14 sessões. As etapas incluíram: entrevista; estabelecimento da linha de base; intervenção (exposição à realidade virtual e avaliações funcionais); encerramento e follow-up após
1 e 3 meses. Os participantes não dirigiam antes do programa de intervenção, e passaram a fazê-lo, tanto no ambiente virtual quanto no não virtual, e relataram redução da ansiedade. Conclui-se que a combinação da exposição à realidade virtual com a avaliação funcional dos comportamentos contribuiu para que os participantes dirigissem mais e com menos medo.

Palavras-chave: terapia de exposição, avaliação funcional, simulador de carro, medo de dirigir, análise do comportamento.

\section{fbstract}

This research evaluated the effects of an intervention program on driving fear, It included functional assessment of behavior and exposure to a virtual reality; the

\footnotetext{
* Elizeu Borloti, Universidade Federal do Espírito Santo, Vitória, Brasil.

** Andressa dos Santos, Verônica Bender Haydu, Universidade Estadual de Londrina, Londrina, Brasil.

Correspondência a respeito deste artigo deve ser endereçada para Verônica Bender Haydu. Correio eletrônico: haydu@uel.br
}

Cómo citar este artículo: Dos Santos, A., Borloti, E. \& Haydu, V. B. (2018). Terapia com exposição à realidade virtual e avaliação funcional para fobia de dirigir: um programa de intervenção. Avances en Psicología Latinoamericana, 36(2), $235-251$. DOI: http://dx.doi.org/10.12804/revistas.urosario.edu.co/apl/a.5329 
capability of the simulator to generate sense of presence and anxiety in participants with fear of driving, and cybersickness. Six adults aged between 22 and 54, who held driving licenses, and complained of fear or phobia participated in the study. A virtual reality driving simulator was used. The procedure consisted of a non-concurrent multiple-baseline design, with 11 to 14 sessions. The stages included interview, baseline establishment, intervention (virtual reality exposure and behavioral assessment), final session and follow-up after one and three months. The participants had not driven before the intervention program and started to do so both in the virtual and in the non-virtual environment, and reported reduced anxiety. The study concluded that the combination of virtual reality exposure and functional assessment of behaviors contributed for the participants to start driving more and with less fear. Keywords: exposure therapy, functional assessment, car simulator, fear of driving, behavior analysis.

\section{Resumen}

Este estudio evaluó los efectos de un programa de intervención en el miedo a conducir. El estudio incluyó la evaluación de: la función de comportamientos de participantes con miedo de conducir; la exposición a la realidad virtual; la capacidad del simulador de generar sensación de presencia y ansiedad; y el cybersickness. Participaron 6 adultos que se quejaban de miedo o fobia a conducir, con edades entre 22 y 54 años, y que poseían pase de conducción. Se utilizó un simulador de conducción de vehículo. El procedimiento consistió en un diseño línea de base múltiple no simultánea, con 11 a 14 sesiones. Los pasos incluyen: entrevista; el establecimiento de la línea base; intervención (exposición a la realidad virtual y evaluaciones funcionales); cerramiento y seguimiento después de 1 y 3 meses. Los participantes no conducían antes del programa de intervención y comenzaron a hacerlo, tanto en el ambiente virtual como en el no virtual, y reportaron una reducción de la ansiedad. Se concluyó que la exposición a la realidad virtual, en combinación con la evaluación conductual-funcional, contribuyó a que los participantes manejaran más y con menos miedo.

Palabras clave: terapia de exposición, evaluación conductual-funcional, simulador de automóvil, miedo a conducir, análisis de la conducta.

Dirigir é uma habilidade essencial para o cidadão contemporâneo. Essa habilidade contribui para a independência e mobilidade necessárias para a participação em diversas atividades cotidianas. Entretanto, $6 \%$ a $8 \%$ da população brasileira que possui Carteira Nacional de Habilitação (CHN) afirma ter medo de dirigir, por estresse pós-traumático ou outros motivos não justificáveis (Cantini et al., 2013). Essa população é caracterizada, em sua maioria, pelo público feminino (Barp \& Mahl, 2013; LeBeau et al., 2010). Para esse público, e também o masculino, o medo e a fobia de dirigir podem acarretar redução da qualidade de vida, gerando prejuízos significativos, como limitar atividades sociais e profissionais. Assim, é importante que sejam desenvolvidas intervenções psicoterapêuticas adequadas e efetivas para o medo e a fobia de dirigir.

No DSM-5 (American Psychiatric Association, 2013), a fobia de dirigir é classificada como sendo um transtorno de ansiedade, uma fobia específica do tipo situacional, que se caracteriza pelo medo ou ansiedade clinicamente significativos (intenso ou grave) diante de um objeto ou situação. $\mathrm{Na}$ presença do estímulo aversivo ou ameaçador (no caso, a situação da direção do carro), uma resposta que o elimina ou adia é emitida pelo indivíduo, o que pode interferir nas atividades de seu dia a dia (Zamignani \& Banaco, 2005). Além disso, o indivíduo pode apresentar respostas incompatíveis com a execução de outras atividades, como as perturbações na coordenação dos movimentos ao volante e aos pedais, e a redução na visibilidade de detalhes dos estímulos ambientais, nos casos de fobia e medo de dirigir (De Araújo, Borloti \& 
Haydu, 2016). Como exemplos adicionais dessas alterações, Cantini et al. (2013) listaram dificuldades em estacionar, em dirigir com trânsito intenso, em sair com o carro em subida e em engrenar as marchas. Além do comportamento de dirigir ser perturbado pela ansiedade, conforme apontaram de Araújo et al., a esquiva da emissão do operante dirigir é mantida pela cessação da aversão do dirigir, e pelo reforço de outros comportamentos, incompatíveis ou não com o dirigir.

No estudo desenvolvido por Cantini et al. (2013), com participantes com CHN, que se esquivavam de dirigir, o medo de dirigir pareceu ser controlado por regras cujas consequências descritas são o erro ao dirigir, a perda do controle do carro, a dificuldade de concentração na via e/ou nos comandos necessários para dirigir e o acidente provocado pelo dirigir. Essas descrições de contingências aversivas também são relatadas por pessoas com CHN que já dirigiram, mas se esquivam de dirigir permanentemente ou ocasionalmente, como em horários com trânsito intenso ou condições climáticas adversas (Costa, de Carvalho, Cantini, Freire \& Nardi, 2014). Assim, pode-se afirmar que as regras apresentadas pelas pessoas com CHN que se esquivam de dirigir são semelhantes, independente de terem tido ou não a experiência de dirigir.

O tratamento de pessoas com fobia geralmente é feito por meio de intervenção psicológica. O processo psicoterapêutico visa promover mudança em determinadas contingências que controlam o comportamento que esteja causando sofrimento, além de proporcionar o desenvolvimento de novos comportamentos. A mudança nas contingências, segundo os princípios da Análise do Comportamento, é viabilizada pela avaliação e análise funcional do comportamento, que envolvem a operacionalização das classes de respostas, dos eventos que antecedem as respostas e das consequências que mantêm essas respostas. A análise funcional visa à manipulação das variáveis mantenedoras do comportamento, pela alteração das contingências de reforço (Sturmey, 1996; Del Prette, 2011).

Além da análise funcional do comportamento, o tratamento envolve a exposição (geralmente graduada) ao estímulo temido (Haydu, Fornazari, Borloti \& Haydu, 2014). O procedimento de exposição ao evento temido foi aperfeiçoado com o desenvolvimento das tecnologias computacionais, como a da realidade virtual (RV). Diversos estudos (e.g., Wald \& Taylor, 2003; Walshe, Lewis, Kim, O'Sullivan \& Wiederhold, 2003) utilizaram a exposição à RV como terapia de participantes com medo ou fobia de dirigir (Costa, Carvalho \& Nardi, 2010). Esses estudos demonstraram que a terapia de exposição por RV (Virtual Reality Exposure Therapy [VRET]) possibilita que o indivíduo se aproxime de situações que ele teme, o que forja contingências para a extinção ou habituação de respondentes e para a aquisição e manutenção de comportamentos alternativos à fuga e à esquiva.

A RV pode ser definida como "um conjunto de estímulos criados por computador que simulam e concorrem com as reais contingências à volta do indivíduo, interagindo com suas respostas e adquirindo controle sobre as mesmas" (Barbosa, 2013, p. 114). Ambientes virtuais possibilitam que o indivíduo seja confrontado com estímulos temidos e, ao mesmo tempo, que se eliciem os respondentes relacionados ao medo e à ansiedade. Os estímulos da RV controlam o comportamento do indivíduo como se ele estivesse presente na realidade não virtual. Esse sentimento de "estar presente" no ambiente virtual é denominado senso de presença (Slater, 2003), um fator importante para a VRET, pois, uma vez que o indivíduo é exposto aos estímulos dos cenários virtuais temidos, espera-se que o mesmo se sinta presente e ansioso, como se sentiria no ambiente não virtual (Ling, Nefs, Morina, Heynderickx \& Brinkman, 2014).

Há um número relativamente pequeno de estudos sobre VRET para medo de dirigir em comparação a estudos sobre outros tipos de medos e 
fobias (Haydu et al., 2016). O primeiro estudo, encontrado na revisão bibliográfica feita por Haydu et al. (2016), foi realizado por Wald e Taylor (2000). Nesse estudo, participou uma mulher com 35 anos, diagnosticada com a fobia específica de dirigir. $\mathrm{O}$ procedimento compreendeu 1 sessão de linha de base, 3 sessões de intervenção, e 3 de follow-up (1, 3 e 7 meses após a intervenção). Observou-se a redução da ansiedade e o aumento no tempo de emissão do dirigir. A melhora clínica manteve-se no follow-up. Wald e Taylor (2003) reapresentaram esse estudo e descreveram um segundo estudo com cinco participantes submetidos à sessões de linha de base, oito sessões de VRET e avaliações em sessões de follow-up (1, 3 e 12 meses após a intervenção). Os resultados mostraram redução de ansiedade e da esquiva em 3 dos 5 participantes.

De acordo com Haydu et al. (2016), há poucos estudos sobre VRET para medo de dirigir e neles "os procedimentos terapêuticos são descritos de forma sucinta, com um número insuficiente de dados para replicações sistemáticas” (p. 79). Assim, visando contornar essa limitação, o presente estudo teve como objetivos: (a) avaliar os efeitos de um programa de intervenção no medo e na fobia de dirigir, que incluiu a exposição a cenários de trânsito por meio de um simulador de RV de veículo automotivo, e a avaliação funcional de comportamentos (análise funcional descritiva); (b) avaliar a capacidade de o simulador gerar senso de presença e ansiedade em participantes com medo de dirigir; e (c) avaliar o mal-estar produzido pela exposição à RV (cybersickness) por meio do simulador.

\section{Método}

\section{Participantes}

Participaram do presente estudo 6 adultos (4 mulheres e 2 homens) com queixa de medo de dirigir e 1 terapeuta (a pesquisadora que conduziu a coleta de dados). A pesquisadora é psicóloga e, na ocasião da coleta, aluna de mestrado, e estava apta a conduzir as sessões de psicoterapia, como analista do comportamento inscrita no Conselho da Classe Profissional de Psicólogos. Ela foi devidamente treinada para programar e operar o simulador.

Os participantes foram selecionados a partir do sistema de triagem de uma Clínica Psicológica ou de convites pessoais da pesquisadora. A idade dos mesmos variou de 22 a 54 anos, e o grau de escolaridade, entre Ensino Médio completo e pós-graduação. Para a seleção foram adotados os seguintes critérios: (a) ter CHN; (b) não ter medo de viajar como passageiro em automóveis; (c) não estar em tratamento psicológico ou farmacológico para controle de ansiedade; (d) não usar substâncias psicoativas de modo abusivo ou dependente; (e) não apresentar distúrbio neurológico do tipo epilepsia ou distúrbio do ouvido do tipo labirinte; e (f) não apresentar escores altos no Simulator Sickness Questionnaire (SSQ) nas sessões de linha de base (50\% das respostas deveriam ser absolutamente não ou levemente em relação ao Cybersickness). Os selecionados assinaram o Termo de Consentimento Livre e Esclarecido, aprovado pelo Comitê de Ética em Pesquisa.

\section{Local, materiais e instrumentos}

As sessões de entrevista e de encerramento ocorreram em uma sala de atendimento da Clínica Psicológica. As sessões das fases de linha de base, intervenção e follow-up de 1 e de 3 meses, com exposição ao simulador de veículo automotivo, aconteceram numa sala da empresa parceira da pesquisa, com condições dignas e apropriadas à natureza da psicoterapia e ao sigilo profissional dela inerente.

Usou-se o Simulador de Realidade Virtual de Veículo Automotivo, desenvolvido pela Oníria LDSoftware S. A., com base nas exigências descritas no Caderno de Requisitos Versão 1.01 do projeto "Estudo do uso de simuladores e recursos de realidade virtual para formação de condutores 
em autoescolas" (Fundação Certi e Fepese - UFSC, 2010). A estrutura física do simulador é composta por sistemas de hardware, de apoio e segurança (banco, cinto de segurança etc.), de comandos (volante, freios, embreagem, acelerador, etc.), e de estruturas elétricas, computacionais, de imagens e de audio. Os recursos de software são formados por: simulação física e dinâmica veicular; síntese gráfica e de som; geração de tráfego; tratamento de entrada/saída; base de terrenos, redes viárias e objetos; editor de configuração da simulação; administrativo e modo de monitoramento. O simulador apresenta um conjunto de cenários urbanos com um traçado aberto, que podem ser navegados de forma contínua. Esses cenários permitem ao usuário interagir com os mesmos comandos e a mesma dinâmica veicular de um carro na exposição in vivo. Os cenários foram os seguintes: $\mathrm{C} 1$ - cenário urbano, sem trânsito, com aumento gradual de pedestres, trajetos com curvas, rotatórias e requisito de paradas em esquinas; $\mathrm{C} 2$ - cenário urbano com trajetos com subidas, com a necessidade de realizar controle simultâneo de embreagem e acelerador em rampas ("teste da rampa") com esquinas; C3 - cenário urbano com presença de pedestres, aumento gradual do trânsito, e trajetos com parada (controle dos pedais), curvas e subidas; C4 - cenário de estacionamento (espaço amplo para estacionar múltiplos veículos, com subidas e semáforo).

Os instrumentos especificados a seguir foram usados para mensurar os comportamentos:

1. Escala de Unidades Subjetivas de Desconforto (Subjective Units of Disconfort Scale [SUDS]) — proposta por Wolpe (1978 [1973])—, composta por uma escala de 10 pontos, cujo valor deve ser respondido oralmente como referência ao nível de ansiedade/desconforto sentido, o qual apresenta boa correlação com outras medidas de ansiedade (Kaplan, Smith \& Coons, 1995);
2. Inventário de Senso de Presença (ISP), desenvolvido por Zacarin, Santos, Perandré \& Haydu (2015), composto por 14 itens, com uma escala Likert de 5 pontos, e especificações sobre os estímulos virtuais e os do ambiente não virtual, reações fisiológicas durante a exposição, e comportamentos emitidos ou eliciados durante a exposição;

3. Simulator Sickness Questionnaire (SSQ), desenvolvido por Kennedy, Lane, Berbaum \& Lilienthal (1993), composto por uma escala Likert de 4 pontos, que avalia o cybersickness que o simulador de RV pode gerar (e.g., desconforto, dor de cabeça, náusea, vertigem, etc.). Uma tradução livre foi feita para este estudo, sendo o resultado corrigido de acordo com Bouchard, Robillard \& Renaud (2007);

4. Driving Cognitions Questionnaire (DCQ), desenvolvido por Ehlers et al. (2007) e adaptado para a população brasileira por Gomes, Lopes, Rossini \& Lopes (2015), composto por 20 itens, com uma escala Likert de cinco pontos, que descrevem situações sobre o indivíduo evitar dirigir. Esse instrumento, segundo Ehlers et al., mostrou boa consistência interna e correlações significativas com outras medidas de fobia de dirigir. O escore total é obtido a partir a soma de cada valor assinalado com um total de 80 pontos (Gomes, Lopes, Rossini \& Lopes, 2015);

5. Diário de Bordo, folhas de registro para a descrição dos episódios (antecedentes, resposta e eventos consequentes) em que enfrentou ou não a situação de dirigir (para a avaliação funcional dos comportamentos, como sintetizado em Rafihi-Ferreira, Santos, Alckmin-Carvalho \& Soares (2016); com especificação da data e hora, a duração dos episódios, se estava acompanhado ou não, a porcentagem da rota que realizou, tendo em vista a que se propôs a realizar, as condições climáticas e de trânsito e o nível de ansiedade sentido; 
6. Roteiro de Entrevista Semiestruturada - com questões sobre a origem e condições que mantinham o medo de dirigir;

7. Checklists - lista dos comportamentos operantes que compuseram a atividade de dirigir e dos que estiveram relacionados com o medo de dirigir.

\section{Procedimento}

A pesquisadora entrou em contato por telefone com os interessados em participar para verificar se eles apresentavam os critérios de inclusão como participantes. Na sessão de entrevista inicial, a pesquisadora descreveu os objetivos e os procedimentos da pesquisa, e em seguida entregou o Termo de Consentimento Livre e Esclarecido para ser assinado. A pesquisadora entrevistou o participante e coletou informações sobre os eventos que contribuíram para que ele se esquivasse de dirigir e quais estímulos dessa situação eliciavam mais ansiedade. Os dados obtidos foram considerados para a seleção da programação dos cenários para as sessões de exposição à RV. Em seguida, foi entregue o DCQ para ser respondido. Ao final da entrevista, foi agendada a sessão seguinte.

O delineamento de linha de base múltipla não simultânea foi utilizado no presente estudo porque os participantes não vinham para as sessões simultaneamente, e nem todos estavam disponíveis quando o estudo foi iniciado. Os participantes foram distribuídos por sorteio em três grupos. $\mathrm{O}$ Grupo 1 (P2 e P6), o Grupo 2 (P3 e P5) e o Grupo 3 (P1 e P4) realizaram, respectivamente, 3, 4 e 5 sessões de linha de base. O programa variou entre 11 e 14 sessões, considerando as sessões de entrevista, linha de base, intervenção, encerramento e follow-up de 1 mês e de 3 meses. Os participantes iniciaram o programa conforme a disponibilidade de cada um.

A fase de linha de base consistiu de 3, 4 ou 5 sessões, de acordo com o grupo. No início das sessões, perguntava-se ao participante sobre como ele estava se sentindo e se vivenciou algo que julgava importante comentar. Para iniciar a exposição, a pesquisadora informou-os sobre a SUDS como descrito a seguir: "Durante a exposição, eu vou perguntar o nível de ansiedade que você está sentido no momento. Assim, você terá que me responder um número de 0 a 10 , caracterizando o seu nível de ansiedade. Lembre-se que zero relaciona-se com completamente relaxado, não há ansiedade, e 10, extremamente ansioso, Ok?”.

A pesquisadora solicitou o nível referente à SUDS durante a exposição aos cenários. Na primeira sessão da fase de linha de base, foi solicitado ao participante a identificação e localização dos componentes básicos da direção do carro. Em seguida, o participante foi exposto ao cenário que simulava o estacionemanto, nas partes planas e sem semáforo, por no máximo $10 \mathrm{~min}$, para que aprendesse os comandos do simulador, relembrando como dirigir. A partir da primeira sessão de linha de base, foram feitos registros, por meio da checklist, dos comportamentos operantes de dirigir e dos comportamentos operantes de medo de dirigir.

Nas sessões de linha de base, seguiram-se as regras de não apontar os erros de condução que o participante emitisse e de não realizar nenhuma intervenção em relação ao comportamento operante de dirigir. A pesquisadora registrou com o uso da checklist, as dificuldades de cada participante, como na posição do pé na embreagem ou dos braços no volante durante o percurso, na noção de espaço para conversões à direita e à esquerda, dentre outras. Esses dados serviram para indicar instruções a serem fornecidas na fase de intervenção.

Após a exposição ao cenário neutro, a pesquisadora convidava o participante a sair do simulador para responder ISP e logo após, o SSQ. Finalmente, foi conduzida uma sondagem, a fim de investigar a experiência com o simulador. Para isso, foram feitas as seguintes perguntas: "O que você sentiu em relação ao simulador?", "Como você se sentiu em relação ao cenário?”, "Há algo que você acredite importante comentar?”. Na última sessão de 
linha de base, a pesquisadora selecionou com cada participante três cenários para serem usados na fase de intervenção. Os cenários foram selecionados a partir das situações que eliciavam ansiedade ao participante e da frequência do contato cotidiano dele com elas.

Na primeira sessão da fase de intervenção, a pesquisadora instruiu o participante a fazer registros no Diário de Bordo, preenchendo a primeira folha junto com ele, referente à semana anterior àquela sessão. A cada sessão, a pesquisadora conduzia uma avaliação funcional junto com o participante, o que era feito dialogando sobre os registros: as oportunidades de ocorrência de episódios comportamentais do dirigir entre as sessões, os eventos antecedentes e consequentes das respostas emitidas e o nível de ansiedade sentido durante cada episódio. Quando não ocorriam comportamentos de dirigir, a pesquisadora questionava sobre o que poderia estar exercendo função para que o participante se esquivasse das situações de dirigir e propunha a ele criar oportunidades para a aproximação a essas situações.

Na primeira sessão de intervenção, a pesquisadora ensinou e praticou com o participante o exercício de respiração diafragmática e deu instruções para ele realizar esse exercício sempre que sentisse, durante a exposição, que seu nível de ansiedade estivesse muito alto. A fase de intervenção foi caracterizada pela exposição aos cenários pré-selecionados, iniciando com cenas que produzem menos medo, indo para aquelas que produzem mais medo. As variáveis manipuladas foram, por exemplo, parar diante de um sinal verde no semáforo e acelerar e parar o trajeto numa subida e, em seguida, dar continuidade ao trajeto.

Durante a exposição aos cenários virtuais, a pesquisadora solicitou o nível de ansiedade (SUDS) pelo menos quatro vezes, conforme a duração da exposição. Após sair do simulador e sentar-se diante de uma mesa, o participante recebeu, sucessivamente, os questionários ISP e SSQ para serem respondidos. No final, a pesquisadora conduziu uma sondagem com as seguintes perguntas: "Como você se sentiu?", "O que você fez para se sentir menos ansioso?", "Como foi dirigir no ambiente virtual?", "Em que momento você se sentiu mais ansioso?", "Você fez o exercício de respiração durante a exposição?". Além disso, a pesquisadora deu feedbacks sobre o comportamento de dirigir no simulador, reforçando os progressos feitos tanto em relação à habilidade de executar determinadas atividades (rampa, conversões, etc.) quanto em relação ao enfrentamento realizado durante a sessão. A partir das informações fornecidas pelo participante e dos dados da checklist, após duas sessões com cada cenário, a pesquisadora decidia sobre o avanço ou não para o próximo cenário, e se havia necessidade de alguma modificação na programação de cenários feita inicialmente.

A sessão de encerramento aconteceu na Clínica Psicológica. A pesquisadora conduziu um diálogo, durante o qual fez as seguintes perguntas ao participante: "Como tem sido para você estar dirigindo?", "O que significa para você apresentar esse comportamento?" e "Quais sentimentos e pensamentos envolvem o dirigir?". Caso o mesmo não respondesse algo relacionado com o que foi apresentado na entrevista inicial (queixa clínica), a pesquisadora fazia perguntas adicionais para obter mais informações. Em seguida, a pesquisadora entregou a folha com as questões de avaliação do programa de intervenção, depois o DCQ para serem respondidos e, finalmente, agradeceu a participação até aquele momento, e agendou as sessões de follow-up.

As sessões de follow-up aconteceram 1 e 3 meses após a última sessão de intervenção. Essas sessões foram iniciadas com um diálogo e com a análise dos registros no Diário de Bordo no período que antecedeu essas sessões. $\mathrm{O}$ participante realizou exercícios de respiração e foi exposto ao último cenário da fase de intervenção. Após a exposição de 20 minutos, o participante foi convidado a sair do simulador para responder os questionários DCQ, ISP e SSQ, sucessivamente. 
No final, a pesquisadora agradeceu mais uma vez a participação na pesquisa.

\section{Resultados}

Na sessão de entrevista, os participantes descreveram ter medo de não conseguir controlar o carro e da avaliação de terceiros. Disseram ainda, que sentiam não estar aptos a dirigir. P2 e P3 relataram que isso ocorria desde a aprovação na prova prática do Detran, e que apresentavam comportamentos emocionais diante da situação de ter que dirigir. P6 informou que, apesar de pilotar uma moto, não conseguia sair do lugar com o carro, e quando conseguia conduzi-lo por um pequeno período, ficava muito ansioso e perdia a concentração, o que o levava a não se comportar como deveria. P1, P3 e P5 relataram ter medo de não conseguir controlar o carro e de não conseguir enfrentar as situações difíceis no trânsito. Afirmaram temer paralisar no trânsito ou fazer alguma "besteira". P4 comentou não ter segurança em dirigir e que se desesperava ao passar por locais onde havia a possibilidade de trânsito intenso, e tinha medo de ser avaliado pelo pai, pelos irmãos ou por outra pessoa no trânsito. Na tabela 1 são apresentados os resumos das queixas, os cenários da exposição e a distribuição dos participantes nos grupos. $\mathrm{Na}$ fase de intervenção, apesar de terem sido previstas seis sessões, houve sessões de intervenção adicionais: duas para P3 e uma para P4, P5 e P6. A adição de sessões ocorreu devido à avaliação da pesquisadora, da necessidade de determinadas habilidades (realizar rampa, rotatória, rampa com parada obrigatória) serem mais bem treinadas ou aos pedidos de refazer algum cenário.

Para avaliar o medo ou fobia de dirigir, utilizou-se o Driving Cognitions Questionnaire (DCQ), de acordo com o qual, quanto maior o escore, maior é a ansiedade e preocupação em relação ao comportamento de dirigir. Os participantes responderam esse instrumento na sessão de entrevista, na de encerramento e na de follow-up de mês 1 e 3 meses. Os dados encontram-se na figura 1 (P5 e P6 não realizaram as sessões de follow-up de 3 meses). Observa-se que os escores de P2, P3, P4, P5 e P6 diminuíram entre as sessões de entrevista e encerramento. P1 não apresentou redução no escore ao se comparar os dados da sessão de entrevista com a de encerramento, mas nas sessões de follow-up houve redução sucessiva. Nas sessões de follow-up de 1 mês, os escores dos participantes

Tabela 1

Distribuição dos participantes nos grupos e cenários a que cada participante foi submetido, e descrição resumida da queixa

\begin{tabular}{|c|c|c|c|c|}
\hline Participantes & Grupos & $\mathrm{N}^{\circ}$ de Sessões adicionais & Queixas relatadas na sessão inicial & Cenários da exposição \\
\hline $\mathrm{P} 1$ & Grupo 3 & - & $\begin{array}{l}\text { Medo de situações ou ocasiões relacionadas ao } \\
\text { dirigir }\end{array}$ & $\mathrm{C} 1, \mathrm{C} 2, \mathrm{C} 3, \mathrm{C} 4$ \\
\hline $\mathrm{P} 2$ & Grupo 1 & - & Sofreu acidente e não conseguia dirigir & $\mathrm{C} 1, \mathrm{C} 2, \mathrm{C} 3$ \\
\hline P3 & Grupo 2 & 2 & $\begin{array}{l}\text { Medo de situações ou ocasiões relacionadas ao } \\
\text { dirigir }\end{array}$ & $\mathrm{C} 1, \mathrm{C} 2, \mathrm{C} 3, \mathrm{C} 4$ \\
\hline P4 & Grupo 3 & 1 & $\begin{array}{l}\text { Avaliação de terceiros - Referente a questões } \\
\text { sociais }\end{array}$ & $\mathrm{C} 1, \mathrm{C} 2, \mathrm{C} 3$ \\
\hline P5 & Grupo 2 & 1 & $\begin{array}{l}\text { Medo de situações ou ocasiões relacionadas ao } \\
\text { dirigir }\end{array}$ & $\mathrm{C} 1, \mathrm{C} 2, \mathrm{C} 3, \mathrm{C} 4$ \\
\hline P6 & Grupo 1 & 1 & $\begin{array}{l}\text { Devido à ansiedade não conseguia realizar a } \\
\text { atividade de dirigir }\end{array}$ & $\mathrm{C} 1, \mathrm{C} 2, \mathrm{C} 3, \mathrm{C} 4$ \\
\hline
\end{tabular}

Obs. Ver especificações das legendas dos cenários no texto. 
diminuíram em relação à sessão de encerramento, com exceção de $\mathrm{P} 2$ e P6, cujos escores em follow-up de 1 mês após a intervenção aumentaram, de 10 para 16 e de 15 para 25 , respectivamente. Nas sessões de follow-up de 3 meses, os escores dos participantes diminuíram em relação a sessão de follow-up de 1 mês.

Na figura 2 estão os escores do Simulator Sickness Questionnaire (SSQ), do Inventário de Senso de Presença (ISP) e da Subjective Units of Disconfort Scale (SUDS), obtidos ao longo das sessões das fases de linha de base e de intervenção e follow up. Os dados foram corrigidos para uma base de 0 a 10. Todos os participantes apresentaram $50 \%$ das respostas em "absolutamente não" e "levemente" de cybersickness (QCS), em todas as sessões de linha de base. A partir do início da intervenção terapêutica, verificaram-se alterações nos escores medidos pelos diferentes instrumentos em comparação à linha de base, conforme descrito a seguir. P1, P2, P3 e P5 apresentaram uma redução do cybersickness da primeira para a segunda sessão de linha de base. Durante a intervenção, de forma geral, os níveis de cybersickness foram baixos (escore 2,0 ou menos). P4 apresentou um aumento na nona sessão (quarta sessão de intervenção) em relação às demais sessões, pontuando escore 2,4 . Ele relatou estar passando por pressões externas e que a parte do cenário em que aumenta a quantidade de vias causou vertigem e incomodo visual. Nas demais sessões, os escores de P4 foram inferiores a 1,0. P5 apresentou os maiores escores de cybersickness em relação aos demais participantes, com exceção da apresentada na nona sessão (quinta sessão de intervenção).

Os escores do ISP apresentam uma variação entre 4,5 a 7,9. Observa-se na figura 2 que: P6 manteve escores entre 6,6 e 7,9 , o que pode ser considerado escore alto em relação aos demais participantes, e P4 apresentou escores 4,5 a 6,8 - os menores escores em comparação aos demais participantes. Os escores das sessões de follow-up se mantiveram relativamente constantes em comparação à última sessão de intervenção em todos os casos. Os participantes relataram que os estímulos e os cenários apresentados eram muito reais e próximos das situações que encontravam no cotidiano, e que isso contribuiu para que enfrentassem as situações de ter que dirigir fora da sessão. Quatro, dos seis participantes (P2, P3, P5

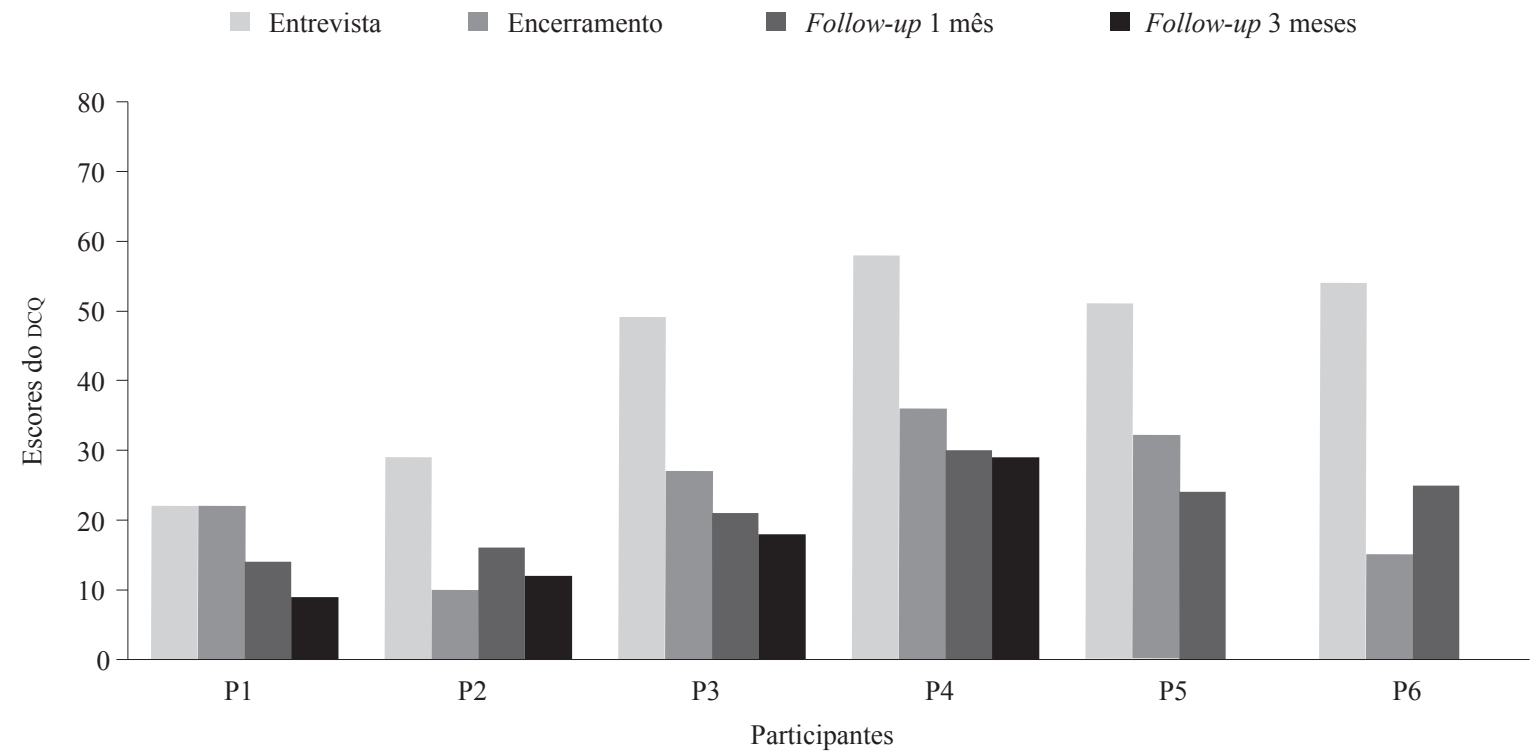

Figura 1. Escores do Driving Congnition Questionnaire (DCQ) 
e P6), relatavam desejar dirigir o carro da família após exposição aos cenários virtuais, com intuito de praticar o que havia ocorrido na sessão.

Em relação a SUDS, P5 reduziu o nível de ansiedade para zero a partir da primeira sessão de linha
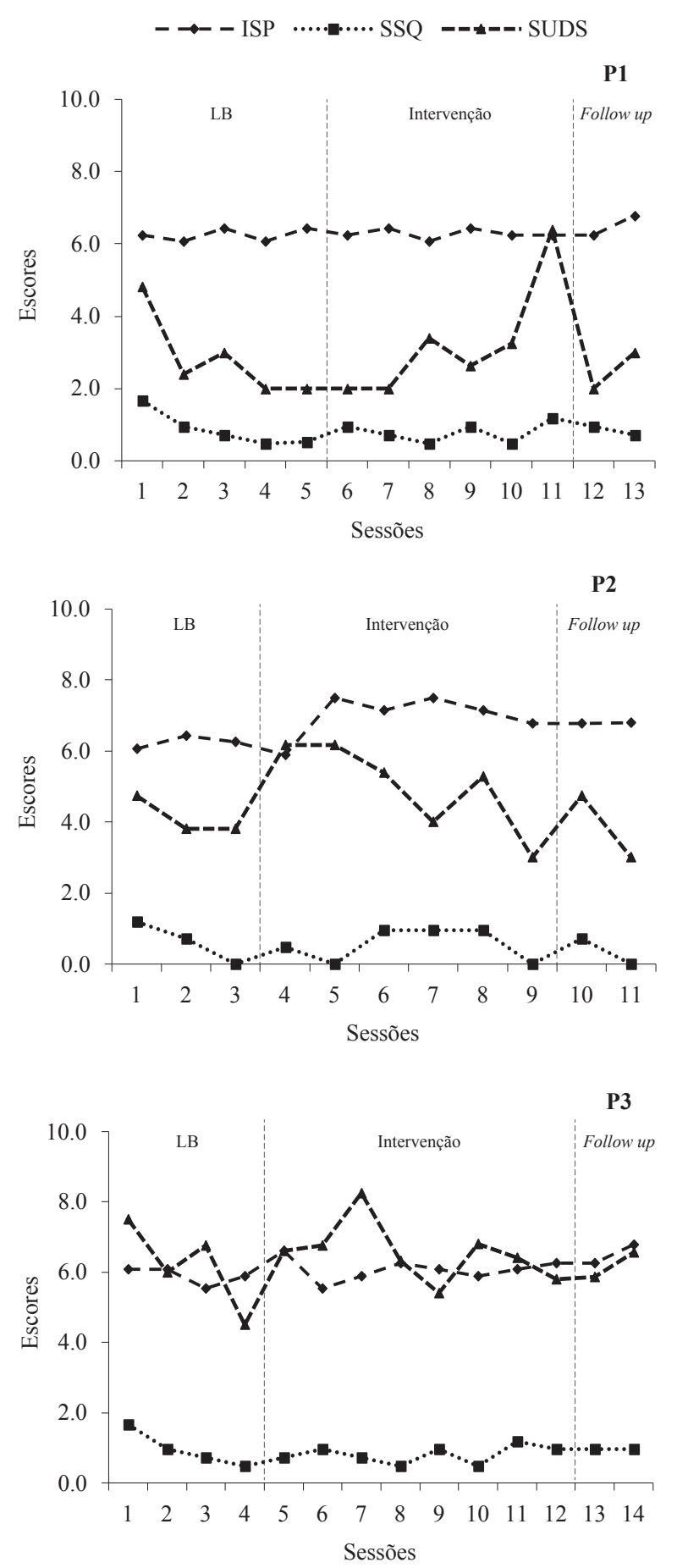

de base, mas da segunda sessão de intervenção em diante, relatou sentir ansiedade (ver figura 2). Os relatos de ansiedade mais intensa para $\mathrm{P} 1, \mathrm{P} 2, \mathrm{P} 3$, P4, P5 e P6, foram observados nas Sessões 11, 5 , $7,6,6,4\left(6^{\mathrm{a}}, 2^{\mathrm{a}}, 3^{\mathrm{a}}, 1^{\mathrm{a}}, 2^{\mathrm{a}}\right.$ e $1^{\mathrm{a}}$ sessões da fase de
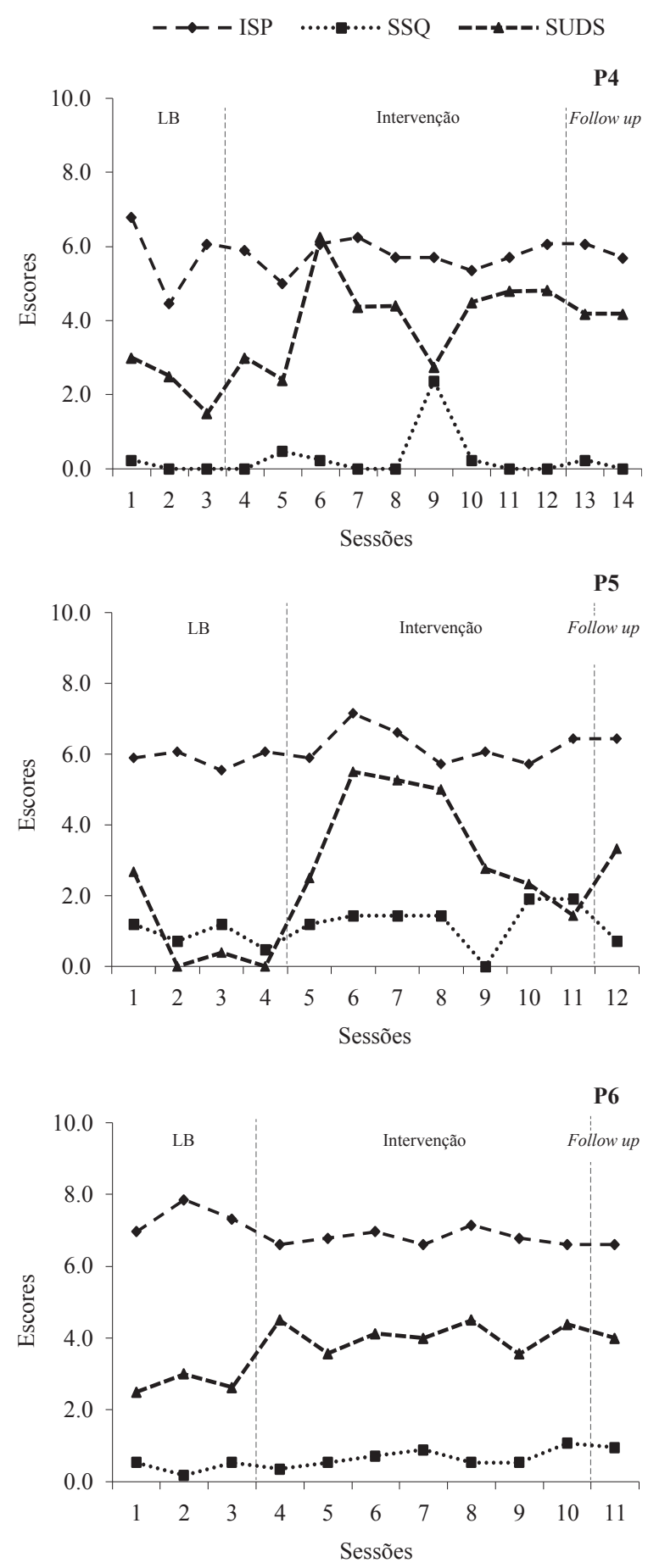

Figura 2. Escores do Simulator Sickness Questionnaire —SSQ-, do ISP e da SUDS por sessão 
intervenção) respectivamente. Na maioria dessas sessões, houve mudança no cenário virtual. Destacam-se os dados de P6, que relatou níveis de ansiedade relativamente constantes nas sessões de linha de base e nas de intervenção, exceto na primeira sessão de intervenção.

Durante as sessões de intervenção, a pesquisadora destacou a importância dos comportamentos adequados para dirigir. Dentre eles, o pisar com o pé direito primeiro no pedal do freio, antes de pisar com o pé esquerdo na embreagem, para realizar a engrenagem da marcha e a redução da velocidade, foi o comportamento emitido com frequência por P4, P5 e P6, mas não por P1, P2 e P3. No entanto, P4 e P6 apresentavam dificuldades para engrenar as marchas: ambos não pisavam o suficiente na embreagem de modo a permitir a troca de marcha. Outro comportamento destacado e modificado por P1, P3 e P5, foi o de segurar o volante na "posição 9 h e 15 min", e mantê-lo nessa posição para controlar a posição do carro na pista, e realizar as curvas com maior precisão. Em quatro sessões da fase de intervenção, P3 demonstrou dificuldade em posicionar o veículo corretamente para conversões abertas à esquerda.

Ainda em relação aos comportamentos operantes relacionados ao medo de dirigir no cenário virtual, todos os participantes passaram por pelo menos uma situação que necessitaram frear bruscamente. Outro operante observado foi pressionar com pouca força o acelerador e deixar o carro "morrer", sendo que nas primeiras sessões de intervenção de todos os participantes isso ocorria na saída para o percurso. $\mathrm{P} 4$ apresentou maior frequência desse modo de pressionar o acelerador durante o trajeto. P1 e P2 deixaram o carro "morrer" com maior frequência nas sessões de mudança de cenário. P1, P2, P3 e P5 demoravam a fazer a troca de marcha, realizando, na maioria das vezes, os trajetos em segunda marcha (isso ocorreu em pelo menos cinco sessões de cada participante). Observou-se que P1 e P2 apresentaram, na maioria das sessões, o comportamento de manter o pé esquerdo na embreagem durante o percurso. P2 afirmou que ao relaxar e sentir-se mais confiante quanto a conduzir o carro, notava que seu pé esquerdo estava na embreagem, retirando-o em seguida.

Nas sessões adicionais de P3, os comportamentos de trocar de marcha, realizar conversão e dirigir na velocidade exigida pela via, modificaram suas propriedades: a latência da troca de marcha diminuiu; a dificuldade em fazer a conversão diminui; e a velocidade do dirigir (inicialmente muito baixa) aumentou, de acordo com a permitida pela via. Na sessão de follow-up de 1 mês, $\mathrm{P} 2$ retirava o pé da embreagem ao ter finalizado a troca de marcha, e na sessão de follow-up de 3 meses não apresentou mais esse comportamento. $\mathrm{O}$ participante que apresentou menor dificuldade ao dirigir em comparação aos demais participantes foi P6.

A partir dos Diários de Bordo dos participantes e das avaliações funcionais realizadas junto com o terapeuta, foi possível verificar que todos tiveram oportunidades e executaram o comportamento de dirigir no dia a dia. P1 e P6 dirigiram em todas as oportunidades. P2, P3, P4 e P5 restringiram-se em dirigir em oportunidades para as quais eles se consideravam mais preparados para enfrentar. $\mathrm{Na}$ figura 3 está a distribuição da frequência de ocasiões que cada participante teve de oportunidades de dirigir e o número de situações enfrentadas durante o programa de intervenção (soma dos resultados dos relatos e das avaliações funcionais de todas as sessões), 1 mês e 3 meses após a sessão de encerramento (relatos e avaliações funcionais realizadas nas sessões de follow-up).

Observa-se na figura 3, que todos os participantes conseguiram realizar pelo menos uma vez a atividade de dirigir durante as sessões de intervenção. Apesar de P3 ter dirigido em todas as oportunidades, relatou que tinha medo de sair dos bairros próximos a sua casa, e que ainda não conseguia ir de carro para o centro da cidade. P2 dirigiu em $63 \%$ das oportunidades e realizou a atividade de dirigir em vários locais, sendo que chegou a levar os pais para um restaurante em um 

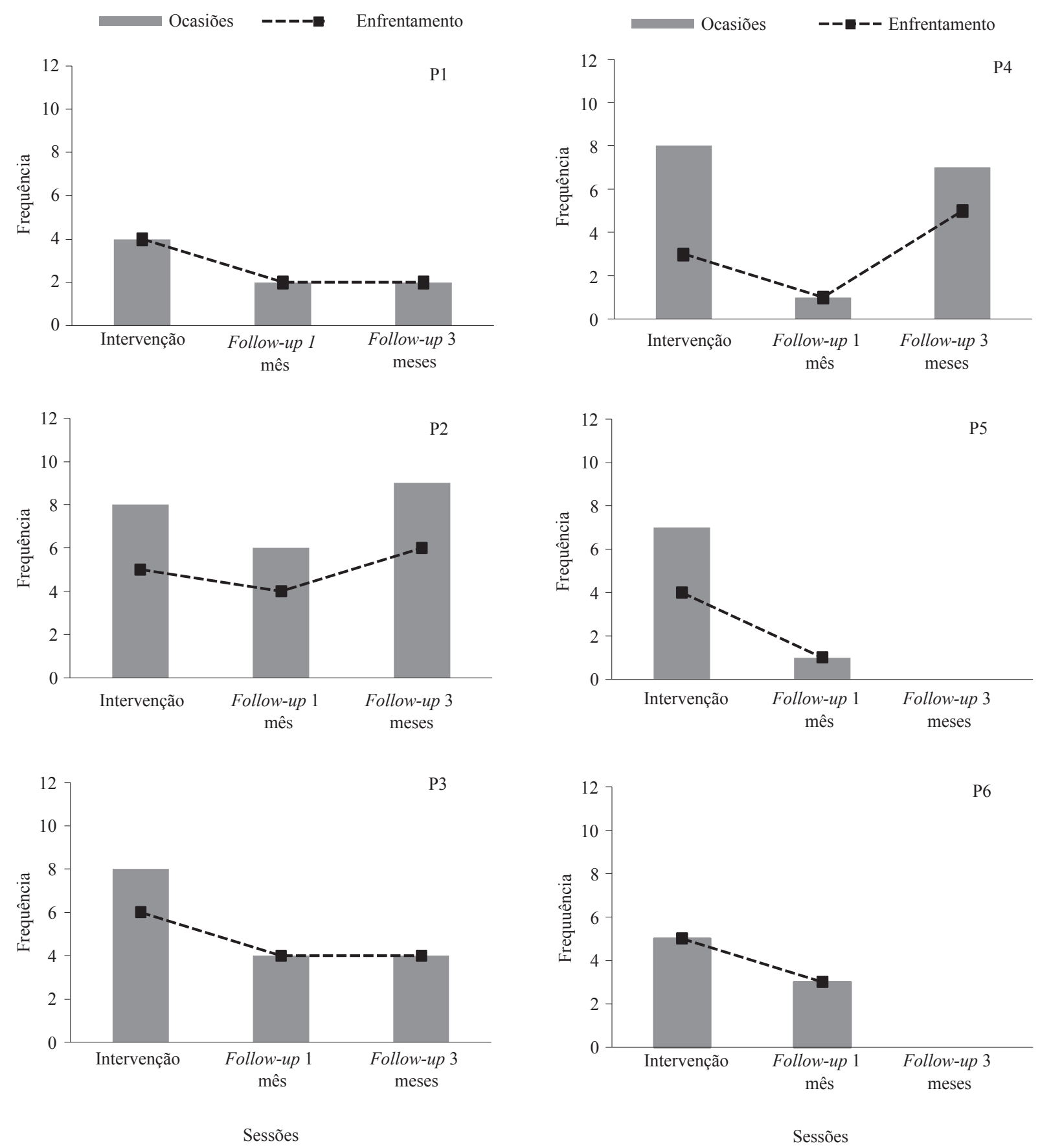

Figura 3. Frequência de ocasiões que cada participante teve oportunidade de dirigir e o número de situações em que dirigiu (enfrentamento)

distrito da cidade (a cerca de $20 \mathrm{~km}$ de distância). P4 e P5 tiveram uma oportunidade de dirigir, sendo que $\mathrm{P} 5$ afirmou estar se programando para renovar a CHN e que seu marido prometeu, ao voltar de viagem, levá-la para treinar dirigir in vivo. Nas últimas sessões, P5, além de ocasionalmente dirigir o carro, muito frequentemente estava pilotando moto. P6 enfrentou todas as oportunidades que surgiram para a atividade de dirigir. P1 relatou maior nível de ansiedade no momento em que 
terminava a atividade. As situações em que P2 enfrentou foram autopropostas, sendo duas após as sessões terapêuticas, em horário de trânsito intenso. As oportunidades que $\mathrm{P} 2$ teve para dirigir, mas não o fez, foram situações não relacionadas com a fobia de dirigir. O nível de ansiedade de P2 e P3 foi reduzindo a cada situação enfrentada. P3 iniciou a atividade de dirigir dentro de quintal espaçoso e a continuou no bairro com trânsito (em todas as situações ela estava sozinha). Ao final da fase de intervenção, P3 conseguiu dirigir a camionete do marido. P4 apresentou o comportamento de dirigir apenas quando algum membro de sua família pedia. As ocasiões que P5 recusou dirigir ocorreram no início do programa de intervenção; ela relatava que não tinha coragem de pedir para dirigir e que ficava com muito medo de pedir e não conseguir conduzir o veículo.

Em relação à avaliação do programa feita pelos participantes, foram destacados a hierarquia dos cenários, o atendimento psicológico com a pesquisadora e o Diário de Bordo como aspectos que contribuíram para que eles colocassem em prática o comportamento de dirigir. P3 e P5 apontaram a necessidade de aumentar o número de sessões de intervenção até que a frequência do comportamento de dirigir aumentasse e mais habilidade fosse adquirida. P1, P2 e P4 sugeriram que o programa deveria compor sessões in vivo com o carro, avaliando que isso contribuiria com o resultado alcançado.

\section{Discussão}

Os objetivos deste estudo estão relacionados a avaliar os efeitos do programa de intervenção proposto, que combinou a avaliação funcional do comportamento com sessões de exposição à realidade virtual por meio de um simulador de carro; e avaliar a tecnologia de realidade virtual como recurso para intervenções terapêuticas no caso do medo ou fobia de dirigir. Para atingir esses objetivos, o estudo iniciou com uma entrevista, na qual os participantes relataram medos de: errar, não conseguir controlar o carro, ser avaliado por terceiros, não estar apto para dirigir, apresentar dificuldades de concentração na via e em relação aos comandos necessários para dirigir, causar acidente, dentre outros. Essa descrição está de acordo com a que foi apresentada no estudo de Cantini et al. (2013), citado anteriormente. Quatro participantes afirmaram não enfrentar as situações de dirigir por não sentir segurança quanto ao controle do carro em situações de trânsito intenso e em algumas condições da via, como a subida.

Em relação à característica da população do presente estudo, tem-se que $67 \%$ (quatro participantes) eram mulheres, em conformidade com a predominância feminina observada em estudos sobre o medo de dirigir (LeBeau et al., 2010; Costa et al., 2010). No presente estudo ocorreu o tratamento de dois participantes do sexo masculino (33\% da população), podendo-se observar que os homens têm reconhecido a necessidade de tratamento para a fobia de dirigir, conforme destacou Cantini et al. (2013).

A maior parte da população com medo ou fobia de dirigir é caracterizada como apresentando esse tipo de transtorno, por ter sofrido acidentes automobilísticos (Costa et al., 2010). Entretanto, no presente estudo apenas uma participante (P2) apresentou essa característica. Isso sugere que o medo de dirigir pode estar relacionado com outros fatores, como crítica social e defasagem na aprendizagem, conforme destacaram Costa et al. (2014) e Barp \& Mahl (2013).

Apenas uma participante do presente estudo tem histórico de acidente, mas mesmo assim, outros participantes (P1, P3 e P5) afirmaram sentir segurança para dirigir no simulador, porque não corriam o risco de causar danos a terceiros. Esse dado confirma avaliação feita por Costa et al. (2010) e por Wiederhold \& Wiederhold (2010), de que dirigir o simulador em local protegido, sem riscos, reduz um possível constrangimento, aumentando a adesão ao tratamento. 
Todos os participantes concordaram que os cenários do simulador proporcionaram ansiedade $\mathrm{e}$ medo como o que sentiam nas condições in vivo, e que os estímulos apresentados no cenário foram suficientes para que eles se sentissem presentes no ambiente virtual. Os resultados no ISP apresentaram estabilidade ao longo das sessões. A importância de os estímulos do cenário eliciarem ansiedade é destacado por autores como Jang et al. (2002) e Walshe et al. (2003), assim como o fato de o usuário poder agir no ambiente virtual do modo como age no ambiente não virtual (McCann et al., 2014). Esses dois aspectos contribuem para que a exposição possa ter efeitos terapêuticos, tanto no que diz respeito à extinção de respondentes quanto para o treino e o aperfeiçoamento do repertório de dirigir, o que foi observado no presente estudo.

Outra medida que possibilitou avaliar o simulador como recurso para ser usado em procedimentos terapêuticos é a obtida pelo SSQ. Em algumas sessões, os participantes relataram desconforto, incomodo visual, dor de cabeça, efeitos semelhantes aos descritos por Beck, Palyo, Winer, Schwagler $\&$ Ang (2007) em um estudo com participantes com medo de dirigir. No presente estudo, o cybersickness foi relatado, principalmente, por P5 no decorrer do programa de intervenção (exceto em uma sessão). De forma geral, os escores do SSQ foram baixos, conforme foi observado também por Bouchard, St-Jacques, Renaud \& Wiederhold (2009), podendo-se sugerir como foi feito por esses autores, que não há motivo para preocupação com a saúde geral e com a segurança dos participantes depois das sessões de exposição à RV. Além disso, os participantes afirmaram que o cybersickness não interferiu com o enfrentamento das cenas que geravam medo de dirigir.

De acordo com o DCQ, usado para medir o medo ou fobia, quanto maior o escore, maior o índice de ansiedade e de preocupação em relação ao comportamento de dirigir. No presente estudo, os participantes apresentaram uma redução do escore após terem passado pelo programa de intervenção, com exceção de P1, que não apresentou alteração entre as sessões de entrevista inicial e de encerramento - o escore apresentado por ela (22) na sessão inicial era o menor em relação aos dos demais participantes. Infere-se que essa participante apresentava uma dificuldade na discriminação da ansiedade relacionada ao dirigir, principalmente antes de iniciar a psicoterapia. Taylor, Deane e Podd (2008) suportam essa inferência ao analisarem a relação entre ansiedade e a habilidade de dirigir, apontando a ocorrência de déficit na habilidade de avaliação e reconhecimento da ansiedade. Nas sessões de follow-up de 1 mês, houve uma redução no escore apresentado por quatro participantes (P1, P3, P4, P5). A exceção foram P2 e P6 que apresentaram escore superior ao da sessão de encerramento, mas na sessão de follow-up de 3 meses, P2 voltou a apresentar redução desse escore. Resultados semelhantes foram observados no estudo de Wald \& Taylor (2003), no qual um dos participantes apresentou escore maior na sessão de follow-up de 1 mês, em relação ao pós-tratamento, e reduziu o escore na sessão de follow-up 3 meses. Os dados dos participantes P3, P4 e P5 são bastante semelhantes aos de Wald e Taylor, havendo redução progressiva nos escores nas sessões de pós-tratamento e de follow-up. Observa-se que esse dado é condizente com os comportamentos de enfrentamento dos participantes, que no início do tratamento não dirigiam, e durante a fase de intervenção passaram a dirigir.

Os participantes tiveram oportunidades de realizar, junto com o terapeuta, avaliações funcionais de seu comportamento de dirigir, observando e verificando os eventos antecedentes e consequentes das suas emissões no dia a dia. Eles descreveram no Diário de Bordo as oportunidades para dirigir durante o período do programa e no começo de cada sessão, e análises de contingências envolvidas nas mesmas foram feitas junto com a terapeuta. Esse procedimento contribuiu para que eles passassem a observar e a descrever as contingências presentes nos momentos em que sentiam medo e ansiedade. 
Assim, verifica-se que os participantes tiveram possibilidade de discriminar as contingências que mantinham as repostas de esquiva e fazer alterações nelas, de forma a terem oportunidade, tanto para treinar o repertório de dirigir quanto para se expor aos estímulos temidos.

Ainda a partir dos dados do Diário de Bordo, verificou-se que P1 teve poucas oportunidades para dirigir, sendo que ela dependia que o filho disponibilizasse o carro, o que pode ter sido um empecilho para o aumento da frequência do comportamento de dirigir. P2, P5 e P6 preferiram dirigir acompanhados de alguém da família. P4 identificou que se opunha a dirigir quando era pressionado ou avaliado por alguém e notou a necessidade de atendimento psicológico para resolução de outras dificuldades.

Os participantes relataram sentir ansiedade durante as exposições no simulador, observada a partir da SUDS e dos comportamentos operantes durante as sessões. Em relação a SUDS, observou-se que não houve redução dos níveis de ansiedade ao se comparar os primeiros cenários de exposição com a última condição de exposição. Isso pode ser devido ao fato de que a última condição de exposição é o cenário classificado como aquele que eliciava mais medo de ser enfrentado. Quanto aos operantes de dirigir, verificou-se que ocorreram com frequência: pressão inadequada de pedais (gerando freada brusca ou parada de motor), demora na troca de marcha e manutenção do pé na embreagem. Essas propriedades de operantes estão relacionadas com o medo e a dificuldade de desenvolver a atividade de dirigir (Detran, 2005), e diminuíram em frequência nas últimas sessões de exposição.

O programa de intervenção apresentou resultados positivos, como o aumento na frequência do comportamento de dirigir. Os participantes relataram que o programa contribuiu para que eles enfrentassem as situações que exigiam dirigir e que isso reduziu o medo que sentiam. Os fatores que contribuíram para isso, segundo eles, foram a exposição à RV, o acompanhamento terapêutico durante as sessões e os registros feitos no Diário de Bordo. Os resultados deste estudo permitem concluir que os participantes passaram a dirigir no dia a dia e que houve redução no nível de ansiedade relatado. Os resultados permitem ainda afirmar que o simulador de veículo automotivo produz senso de presença e elicia respostas de ansiedade, e que é um recurso apropriado para intervenções em caso de medo ou fobia de dirigir. A avaliação funcional do comportamento de dirigir foi fundamental para que os participantes discriminassem as variáveis que afetavam seus comportamentos e para que aprendessem formas alternativas de se comportar. A partir dos resultados sugere-se que, em pesquisas futuras, sejam feitos estudos comparativos em que o programa aqui proposto seja comparado com outros programas de intervenção.

\section{Referências}

American Psychiatry Association (2013). Diagnostic and statistical manual of mental disorders - DSM-5 (5 ed). Washington: American Psychiatric Association.

Barbosa, J. I. C. (2013). Terapia por realidade virtual (VRET): uma leitura analítico-comportamental. Boletim Contexto, 38, 113-131.

Barp, M. \& Mahl, A. C. (2013). Amaxofobia: um estudo sobre as causas do medo de dirigir. Unoesc \& Ciência - ACBS, 4, 39-48. Recuperado de http://editora.unoesc.edu.br/index.php/acbs/ article/view/2627

Beck, J. G., Palyo, S. A., Winer, E. H., Schwagler, B. E. \& Ang, E. J. (2007). Virtual reality exposure therapy for PTSD symptoms after a road accident: an uncontrolled case series. Behavior Therapy, 38(1), 39-48. DOI: 10.1016/j.beth.2006.04.001

Bouchard, S., Robillard \& Renaud, P. (2007). Revising the factor structure of the Simulator Sickness Questionnaire. Annual Review of 
CyberTherapy and Telemedicine, 5, 117-122.

Recuperado de http://w3.uqo.ca/cyberpsy/docs/ qaires/ssq/2007ssq.pdf

Bouchard, S., St-Jacques, J., Renaud, P. \& Wiederhold, B. K. (2009). Side effects of immersions in virtual reality for people suffering from anxiety disorders. Journal of Cybertherapy and Rehabilitation, 2, 127-137. Recuperado de https:// www.highbeam.com/doc/1G1-225437133.html

Cantini, J. A., Ribeiro, L., Andrade, S. M. H. P., Pereira, V. M., Nardi, A. E. \& Silva, A. C. (2013). Medo e evitação na direção de veículos: características de motoristas que nunca dirigiram após obtenção da Carteira Nacional de Habilitação. Jornal Brasileiro de Psiquiatria, 62, 124-130. DOI: 10.1590/s0047-20852013000200005

Costa, R. T., Carvalho, M. R. \& Nardi, A. E. (2010). Exposição por realidade virtual no tratamento do medo de dirigir. Psicologia: Teoria e Pesquisa, 26(1), 131-137. DOI: 10.1590/S010237722010000100015

Costa, R. T., de Carvalho, M. R., Cantini, J., Freire, R. C. R. \& Nardi, A. E. (2014). Demographics, clinical characteristics and quality of life of Brazilian women with driving phobia. Comprehensive Psychiatry, 55, 374-379. DOI: 10.1016/j.comppsych.2013.08.003

De Araújo, A. H., Borloti, E. \& Haydu, V. B. (2016). Ansiedade em provas: um estudo na obtenção da licença para dirigir. Psicologia Ciência e Profissão, 36, 1-14. DOI: 10.1590/19823703000592014

Del Prette, G. (2011). Treino didático de análise de contingências e previsão de intervenções sobre as consequências do responde. Perspectivas em Análise do Comportamento, 2, 53-71. Recuperado de https://docs.wixstatic.com/ugd/89bfd6_053930ea84144de0a8a563492952e13e.pdf

Detran (2005). Direção defensiva: trânsito seguro é direito de todos. São Paulo: Fundação Carlos Chagas.

Ehlers, A., Taylor, J. E., Ehring, T., Hofmann, S. G., Deane, F. P., Roth, W. T. \& Podd, J. V. (2007).
The driving cognitions questionnaire: development and preliminary psychometric properties. Journal of Anxiety Disorders, 21, 493-509. DOI: 10.1016/j.janxdis.2006.08.002

Fundação Certi e Fepese - UFSC (2010). Caderno de Requisitos Versão 1.01 do projeto: Estudo do uso de simuladores e recursos de realidade virtual para formação de condutores em autoescolas. Florianópolis: UFSC.

Gomes, I. C. O., Lopes, E. J., Rossini, J. C. \& Lopes, R. F. F. (2015). Propriedades psicométricas da versão brasileira do Driving Cognitions Questionnaire DCQ. Avaliação Psicológica, 14(3), 319-327. DOI: 10.15689/ap.2015.1403.03

Haydu, V. B., Fornazari, S. A., Borloti, E. B. \& Haydu, N. B. (2014). Facetas da exposição in vivo e por realidade virtual na intervenção psicológica no medo de dirigir. Psico (PUCRS), 4, 136-146.

Haydu, V. B., de Paula, M. B., Zacarin, M. R., Santos, A., Borloti, E. \& Fornazari, S. A. (2016). Terapia por meio de exposição à realidade virtual para medo e fobia de dirigir: uma revisão de literatura. Avances en Psicología Latinoamericana, 34(1), 67-81. DOI: 10.12804/apl34.1.2016.05

Jang, D. P., Kim, I. Y., Nam, S. W., Wiederhold, B. K., Wiederhold, M. D. \& Kim, S. I. (2002). Analysis of physiological response to two virtual environments: driving and flying simulation. CyberPsychology \& Behavior, 5(1), 11-18. DOI: 10.1089/109493102753685845

Kaplan, D. M., Smith, T. \& Coons, J. (1995). A validity study of the subjective unit of discomfort (SUD) score. Measurement and Evaluation in Counseling and Development, 27, 195-199. DOI: 10.1891/1933-3196.2.1.57

Kennedy, R. S., Lane, N. E., Berbaum, K. S. \& Lilienthal, M. G. (1993). Simulator Sickness Questionnaire: an enhanced method for quantifying simulator sickness. International Journal of Aviation Psychology, 3(3), 203-220. DOI: 10.1207/s15327108ijap0303_3

LeBeau, R. T., Glenn, D., Liao, B., Wittchen, H.-U., Beesdo-Baum, K., Ollendick, T. \& Craske, M. 
G. (2010). Specific phobia: A review of DSM-IV specific phobia and preliminary recommendations for DSM-V. Depression and Anxiety, 27, 148-167. DOI: $10.1002 /$ da.20655

Ling, Y., Nefs, H. T., Morina, N., Heynderickx, I. \& Brinkman W. P. (2014). A meta-analysis on the relationship between self-reported presence and anxiety in virtual reality exposure therapy for anxiety disorders. PLOS ONE, 9, e96144. DOI: 10.1371/journal.pone.0096144

McCann, R. A., Armstrong, C. M., Skopp, N. A., Edwards-Stewart, A., Smolenski, D. J., June, J. D., Metzger-Abamukong, M. \& Reger, G. M. (2014). Virtual reality exposure therapy for the treatment of anxiety disorders: an evaluation of research quality. Journal of Anxiety Disorders, 28, 625-631. DOI: 10.1016/j. janxdis. 2014.05.010

Rafihi-Ferreira, R., Santos, D. R., Alckmin-Carvalho, F. \& Soares, M. R. Z. (2016). Clínica analítico-comportamental: histórico, treinamento e supervisão. Perspectivas em Análise do Comportamento, 7, 183-196. DOI: 10.18761/ pac.2016.001

Slater, M. (2003). A note on presence terminology. Presence Connect, 3(3), 1-5.

Sturmey, P. (1996). Functional analysis in clinical psychology. Chichester: John Wiley \& Sons.

Taylor, J., Deane, F. P. \& Podd, J. (2008). The relationship between driving anxiety and driving skill: A review of human factors and anxiety-performance theories to clarify future research needs. New Zealand Journal of Psychology, 37, 28-37. Recuperado de http://www.psychology. org.nz/wp-content/uploads/NZJP-Vol371-20084-Taylor.pdf

\section{Recebido: janeiro 18, 2017} Aprovado: julho 23, 2017
Wald, J. \& Taylor, S. (2000). Efficacy of virtual reality exposure therapy to treat driving phobia: A case report. Journal of Behavior Therapy and Experimental Psychiatry, 31(4), 249-257. DOI: 10.1016/S0005-7916(01)00009-X

Wald, J. \& Taylor, S. (2003). Preliminary research on the efficacy of virtual reality exposure therapy to treat driving phobia. CyberPsychology \& Behavior, 6, 459-465. DOI: 10.1089/109493103769710488

Walshe, D. G., Lewis, E. J., Kim, S. I., O’Sullivan, K. \& Wiederhold, B. K. (2003). Exploring the use of computer games and virtual reality in exposure therapy for fear of driving following a motor vehicle accident. CyberPsychology \& Behavior, 6(3), 329-334. DOI: 10.1089/109493103322011641

Wiederhold, B. K. \& Wiederhold, M. D. (2010). Virtual reality treatment of posttraumatic stress disorder due to motor vehicle accident. Cyberpsychology, Behavior, and Social Networking, 13, 21-27. DOI: $10.1089=$ cyber.2009.0394

Wolpe, J. (1978 [1973]). Prática da terapia comportamental ( $2^{\mathrm{a}}$ ed.) W. G. Clark Jr. (trad.). São Paulo: Brasiliense.

Zacarin, M. R. J., Santos, A., Perandré, Y. R. T. \& Haydu, V. B. (2015). Inventário de senso de presença. Anais do $4^{\circ}$ Seminário em Análise do Comportamento: pesquisas experimentais, translacionais e aplicadas (p. 13). Londrina: UEL.

Zamignani, D. R. \& Banaco, A. R. (2005). Um panorama analítico-comportamental sobre os transtornos de ansiedade. Revista Brasileira de Terapia Comportamental e Cognitiva, 7, 77-92. 